\title{
Determinantes socioeconómicos de salud y COVID-19 en
} México

\author{
Luis A. Chávez-Almazán, ${ }^{1}$ Lorena Díaz-González ${ }^{2 *}$ y Mauricio Rosales-Rivera ${ }^{2}$ \\ ${ }^{1}$ Banco de Sangre Regional Zona Centro, Secretaría de Salud de Guerrero, Guerrero; ${ }^{2}$ Centro de Investigación en Ciencias, Universidad Autónoma
} del Estado de Morelos, Cuernavaca, Morelos. México

\section{Resumen}

Introducción: La población en situación de pobreza presenta una carga patológica mayor que los estratos sociales con mejores posibilidades económicas. Objetivo: Determinar la influencia de los factores socioeconómicos y demográficos en la morbilidad, mortalidad y letalidad de COVID-19 en municipios y estados de México. Métodos: Se analizó la morbilidad, mortalidad y letalidad asociadas a COVID-19 conforme el índice de desarrollo humano y sus indicadores, así como el tipo de población. Se realizaron análisis estadísticos descriptivos, de correlación entre variables de desarrollo versus morbilidad, mortalidad y letalidad, pruebas de asociación y agrupaciones jerárquicas. Resultados: Se observaron correlaciones positivas entre la morbilidad y mortalidad y el índice de desarrollo humano; la letalidad por COVID-19 aumentó conforme disminuyó dicho índice. Existió riesgo significativamente superior de alta letalidad en las localidades con moderado y bajo desarrollo, así como en aquellas con menos de 49999 habitantes. Los principales factores asociados a la letalidad fueron la falta de acceso a los servicios de salud, la vulnerabilidad por ingreso y la carencia social. Conclusiones: La evidencia generada debe llevar a decisiones tendentes al mejoramiento de la calidad de vida de la población con carencias y vulnerabilidades sociales, que necesita ser protegida contra las consecuencias de la actual pandemia de COVID-19.

PALABRAS CLAVE: Marginación. SARS-CoV-2. México. Cobertura de salud. COVID-19.

\section{Socioeconomic determinants of health and COVID-19 in Mexico}

\section{Abstract}

Introduction: The population living in conditions of poorness has a heavier pathological burden than social strata with better economic possibilities. Objective: To determine the influence of socioeconomic and demographic factors on COVID-19 morbidity, mortality and lethality in municipalities and states of Mexico. Methods: Morbidity, mortality and lethality associated with COVID-19 were analyzed according to the human development index and its indicators, and type of population. Descriptive statistical analyses, correlations between developmental variables and morbidity, mortality and lethality, association tests and hierarchical groupings were conducted. Results: Positive correlations were observed between morbidity and mortality and the human development index; COVID-19 fatality increased as the values of said index decreased. There was a significantly higher risk of elevated mortality in localities with moderate and low development, and in those with less than 49,999 inhabitants. The main factors associated with fatality were lack of access to health services, income vulnerability and social deprivation. Conclusions: The evidence generated should lead to decisions aimed at improving the quality of life of the population with social deprivations and vulnerabilities, which needs to be protected against the consequences of current COVID-19 pandemic.

KEYWORDS: Marginalization. SARS-CoV-2. Mexico. Health coverage. COVID-19.

Correspondencia:

*Lorena Díaz-González

E-mail: Idg@uaem.mx
Fecha de recepción: 17-05-2021

Fecha de aceptación: 27-05-2021

DOI: $10.24875 /$ GMM.21000302
Gac Med Mex. 2022;158:4-11

Disponible en PubMed

www.gacetamedicademexico.com

CC BY-NC-ND (http://creativecommons.org/licenses/by-nc-nd/4.0/). 


\section{Introducción}

Los determinantes sociales de la salud están definidos por la Organización Mundial de la Salud como el conjunto de factores socioeconómicos, políticos y estructurales que confluyen en una sociedad determinada. Sumados a las características de los individuos, la susceptibilidad o resistencia a las enfermedades y la accesibilidad a la atención médica de calidad, dichos determinantes producen diferentes resultados en la salud. En México, en 2018, de acuerdo con cifras del Consejo Nacional de Evaluación de la Política de Desarrollo Social (Coneval), $41.9 \%$ de la población se encontraba en situación de pobreza, es decir, aproximadamente 52.4 millones de personas. Ese amplio sector de la población carece de la posibilidad de acceder a un nivel de bienestar aceptable, lo que le confiere vulnerabilidad y riesgo ante enfermedades y desastres naturales, entre otros.

La actual pandemia de COVID-19 significa un reto para los sistemas de salud, el cual implica dar cobertura a todos los ciudadanos que necesiten atención médica y brindarles los medios adecuados para superar la enfermedad y evitar el fallecimiento. Sin embargo, en México existen barreras importantes que impiden lograr este reto, como la marginación, la pobreza y la falta de acceso a los servicios de salud. Los efectos de la pandemia pueden evidenciar las carencias y necesidades básicas de la población, tales como la alimentación, ingreso igual o apenas superior a la línea de bienestar y vivienda digna. La población en situación de pobreza presenta una carga patológica mayor que los estratos de la sociedad con mejores posibilidades económicas. ${ }^{1}$ En este sentido, la pobreza y la salud deficiente están interrelacionadas, es decir, mientras exista la pobreza se obtendrán peores resultados de salud y, a su vez, la salud deficiente generará más pobreza. ${ }^{2}$

Además, la población de México enfrenta condiciones adversas, como la alta prevalencia de obesidad, diabetes y enfermedades cardiovasculares, precariedad laboral, problemas de acceso al agua y hacinamiento, que impiden la adopción generalizada de medidas preventivas y múltiples brechas de acceso a derechos sociales como la salud, la alimentación, la educación, la vivienda y la seguridad social. ${ }^{3-6}$ Se ha reportado que la alta prevalencia de la pobreza, aunada a las altas tasas de enfermedades crónicas como la diabetes y la obesidad, significó que las muertes no se limitaran a la población anciana de alto riesgo. ${ }^{7}$
En ese contexto, se ha concluido que los países en desarrollo, como México, la pobreza y la desigualdad generalizadas han alimentado la pandemia de COVID-19 y es probable que empeoren como consecuencia. ${ }^{8}$ Además, se ha mencionado que esta crisis sanitaria afectará gravemente a los grupos de población más vulnerables y puede revertir los avances en materia de desarrollo social de la última década y comprometer la capacidad de recuperación económica de los hogares. ${ }^{6}$

Por lo anterior, es necesario que se genere evidencia sobre el grado de afectación de COVID-19 a la gente más pobre y vulnerable. Esta información podría ser de utilidad para los tomadores de decisiones en el desarrollo de estrategias tendentes a la protección de la salud de estos sectores sociales.

En este trabajo transversal se determinará la influencia de los factores socioeconómicos sobre la morbilidad, mortalidad y letalidad de COVID-19 en municipios, estados o regiones de la República Mexicana.

\section{Métodos}

Se obtuvieron los datos abiertos de la Dirección General de Epidemiología de la Secretaría de Salud (https://datos. covid-19.conacyt.mx/) sobre casos confirmados y muertes asociadas a COVID-19. El procesamiento de la información se realizó en Python ${ }^{9,10}$ de la siguiente forma:

- Se calcularon las proporciones de morbilidad, mortalidad y letalidad municipales y estatales.

$$
\begin{gathered}
\text { Morbilidad }=(\text { casos confirmados/tamaño de } \\
\text { población }) \times 100000 \\
\text { Mortalidad }=(\text { fallecidos/tamaño de } \\
\text { población }) \times 100000
\end{gathered}
$$

Letalidad $=($ fallecidos $/$ (casos confirmados $) \times 100$

- A cada municipio fue añadido el índice de desarrollo humano (IDH) y los porcentajes de los 16 indicadores socioeconómicos desarrollados por el Coneval (https://www.coneval.org.mx/Medicion/Paginas/Programas_BD_municipal.aspx): ${ }^{11}$

1. Pobreza

2. Pobreza extrema.

3. Pobreza moderada.

4. Vulnerabilidad por carencia social.

5. Vulnerabilidad por ingreso.

6. No pobre y no vulnerable.

7. Rezago educativo.

8. Carencia por acceso a los servicios de salud.

9. Carencia por acceso a la seguridad social.

10. Carencia por calidad y espacios de la vivienda. 

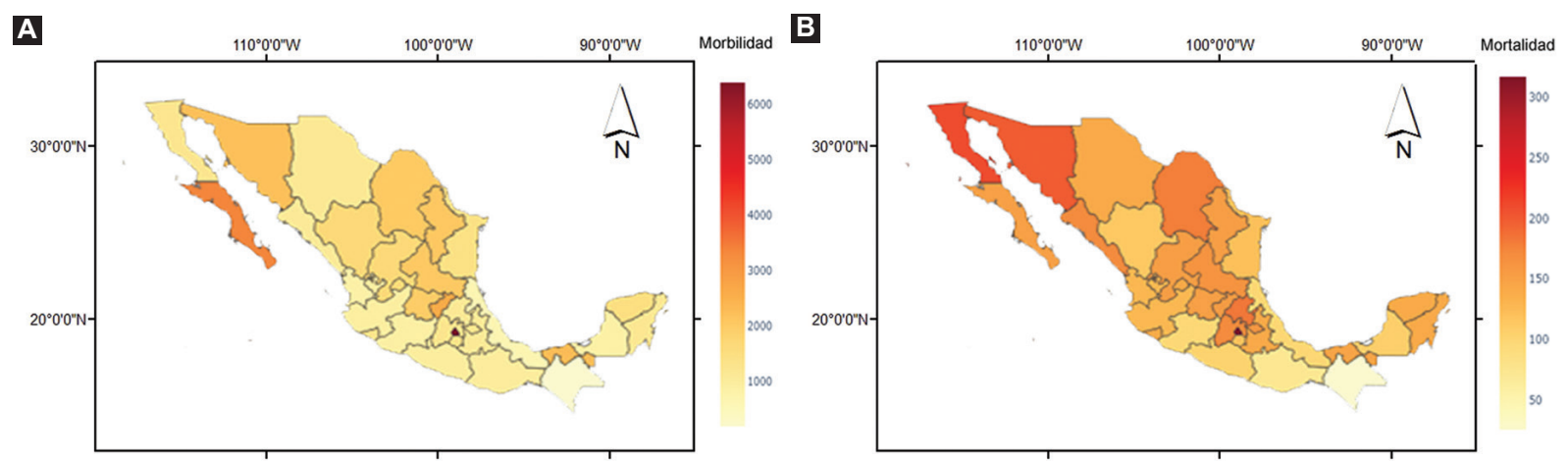

C

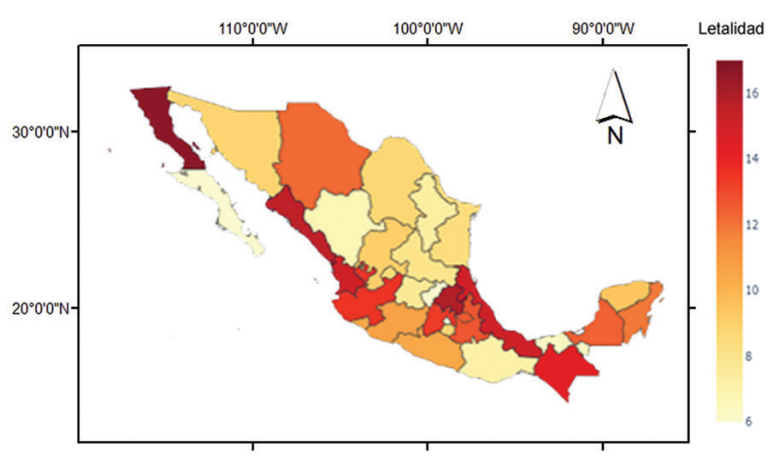

Figura 1. Mapas de morbilidad, mortalidad y letalidad de COVID-19 en los estados de México, con información actualizada el 10 de marzo de 2021.

11. Carencia por acceso a los servicios básicos en la vivienda.

12. Carencia por acceso a la alimentación.

13. Población con al menos una carencia social.

14. Población con tres o más carencias sociales.

15. Población con ingreso inferior a la línea de bienestar.

16. Población con ingreso inferior a la línea de bienestar mínimo.

A nivel municipal se crearon las variables categóricas de nivel de IDH muy alto (0.800-1.000), alto (0.700$0.799)$, moderado $(0.551-0.699)$ y bajo $(<0.550)$. Por su parte, las localidades fueron categorizadas de acuerdo con su número de habitantes: ${ }^{12}$

- Zonas metropolitanas grandes (1 000000 o más habitantes).

- Ciudades medianas (250 000-999 999 habitantes).

- Ciudades pequeñas (50 000-249 999 habitantes).

- Localidades semiurbanas (10 000-49 999 habitantes).

- Poblaciones semirrurales (2500-9999 habitantes).

- Poblaciones rurales (menos de 2500 habitantes).

\section{Análisis estadístico}

- Regresión lineal ordinaria para explorar relaciones bivariadas ${ }^{13}$ del IDH con la morbilidad, mortalidad y letalidad.
- Razón de momios (RM) para determinar el riesgo de los municipios de presentar alta morbilidad, mortalidad y letalidad (proporciones menores y mayores que la media nacional), con base en el IDH (muy alto y alto versus moderado y bajo) y el tamaño de su población $(<2500$ 49999 habitantes versus 50000 a > 1000000 habitantes).

- Análisis de conglomerados jerárquico,,$^{14,15}$ con el empleo de la distancia euclidiana máxima para explorar en forma multivariada las siguientes variables: tasas de morbilidad, mortalidad y letalidad y los 16 indicadores socioeconómicos.

- Algoritmo t-Distributed Stochastic Neighbor Embedding (t-SNE) a través del programa Orange, ${ }^{16}$ para la visualización de los datos. Se usaron las siguientes variables: tasas de morbilidad, mortalidad y letalidad, así como IDH y medidas de pobreza multidimensional.

\section{Resultados}

Al 10 de marzo de 2021, en el mundo se reportaron 118095307 casos (Figura 1A) y 2620776 defunciones (Figura 1B) por COVID-19, con una tasa de letalidad de $2.2 \%$. Los países que concentraron la mayor cantidad de casos confirmados 
Tabla 1. Países con mayor cantidad de casos confirmados y defunciones por COVID-19

\begin{tabular}{|c|c|c|c|c|}
\hline $\begin{array}{l}\text { Posición } \\
\text { mundial }\end{array}$ & País & $\begin{array}{c}\text { Casos } \\
\text { confirmados }\end{array}$ & $\begin{array}{c}\text { Casos } \\
\text { defunciones }\end{array}$ & Letalidad \\
\hline 1 & $\begin{array}{l}\text { Estados } \\
\text { Unidos }\end{array}$ & 29224389 & 530173 & 1.81 \\
\hline 2 & India & 11285561 & 158189 & 1.40 \\
\hline 3 & Brasil & 11202305 & 270656 & 2.42 \\
\hline 4 & Rusia & 4302726 & 88773 & 2.06 \\
\hline 5 & Inglaterra & 4247879 & 125222 & 2.95 \\
\hline 6 & Francia & 4022429 & 89707 & 2.23 \\
\hline 7 & España & 3172101 & 71961 & 2.27 \\
\hline 8 & Italia & 3123368 & 100811 & 3.23 \\
\hline 9 & Turquía & 2821943 & 29227 & 1.04 \\
\hline 10 & Alemania & 2541781 & 72858 & 2.87 \\
\hline 11 & Colombia & 2285960 & 60773 & 2.66 \\
\hline 12 & Argentina & 2169694 & 53359 & 2.46 \\
\hline 13 & México & 2144486 & 192491 & 8.98 \\
\hline 14 & Polonia & 1828313 & 45997 & 2.52 \\
\hline
\end{tabular}

(71.4\% del total global), se describen en la Tabla 1. México ocupó la posición 13 con 2144486 casos confirmados y la número tres en defunciones (192 491 casos), con una letalidad de $8.98 \%$ (Figura 1C). ${ }^{17}$

\section{Análisis de COVID-19 en los estados de la República Mexicana}

La Ciudad de México, Baja California Sur y Querétaro presentaron proporciones de casos confirmados muy altas: 6392,3354 y 2623 casos $/ 100000$ habitantes, respectivamente. Por otro lado, algunos estados no rebasaron los 1000 casos/100 000 habitantes, como Guerrero, Jalisco, Michoacán, Campeche, Nayarit, Veracruz y Chiapas. La proporción de mortalidad también fue alta en la Ciudad de México (317 muertes/100 000 habitantes), Baja California y Sonora, con 210 y 199 defunciones/100 000 habitantes, respectivamente. Chiapas presentó la menor proporción de mortalidad, con 25 defunciones/100 000 habitantes, así como Oaxaca, Michoacán y Veracruz, con $<100 / 100000$ habitantes (Figura 1).

\section{Correlación entre indicadores epidemiológicos y el índice de desarrollo humano}

Se observaron correlaciones positivas entre las proporciones de morbilidad $(r=0.536)$ y mortalidad $(r=0.538)$ respecto al IDH de los municipios (Figuras $2 \mathrm{~A}-\mathrm{C}$ ). Dichas correlaciones fueron más altas a nivel estatal: $r=0.741$ en morbilidad y $r=0.812$ en mortalidad (Figuras 2D-E). Por el contrario, la letalidad mostró una tendencia negativa, la cual fue más acentuada en los estados $(r=-0.289)$ que en los municipios $(r=-0.095)$ (Figuras 2C-F).

\section{Razón de momios (Tabla 2)}

a) Índice de desarrollo humano. Los municipios con mejor IDH (muy alto y alto) presentaron mayor riesgo de morbilidad $(\mathrm{RM}=6.58, \mathrm{IC}=5.02-8.62)$ y mortalidad $(\mathrm{RM}=7.82, \mathrm{IC}=6.05-10.13)$ por arriba de la media nacional. En contraste, los municipios con moderado y bajo IDH tuvieron una importante protección hacia estos indicadores epidemiológicos $(\mathrm{RM}=0.15, \mathrm{IC}=0.12-0.19$ en morbilidad y $\mathrm{RM}=0.13, \mathrm{IC}=0.09-0.17$ en mortalidad). Los municipios con alto y muy alto IDH tuvieron protección de presentar letalidad por arriba de la media nacional $(\mathrm{RM}=0.79$, $\mathrm{IC}=0.64-0.98$ ), mientras que los municipios con bajo y moderado IDH presentaron riesgo de alta letalidad (RM = 1.26, IC = 1.02-1.56).

b) Tamaño de población. Se encontró que las localidades menores a 49999 habitantes presentaron, por un lado, protección para tener baja morbilidad y mortalidad por COVID-19 y, por el otro, aumento del riesgo significativamente para la letalidad $(\mathrm{RM}=1.54, \mathrm{IC}=1.22-1.94)$. Las localidades mayores a 50000 habitantes presentaron menor riesgo de alta letalidad ( $\mathrm{RM}=0.65, \mathrm{IC}=0.51-0.82$ ).

c) Densidad poblacional. Se observó asociación entre la densidad poblacional y la morbilidad y mortalidad por COVID-19: existió alto riesgo para estos indicadores epidemiológicos en las poblaciones con alta densidad (morbilidad, $\mathrm{RM}=4.20$, $\mathrm{IC}=3.09-5.69$; mortalidad, $\mathrm{RM}=5.98, \mathrm{IC}=4.28$ 8.36) y con densidad menor que la media nacional se observó protección estadísticamente significativa para morbilidad $(\mathrm{RM}=0.24, \mathrm{IC}=$ $0.18-0.32)$ y mortalidad $(\mathrm{RM}=0.17, \quad \mathrm{IC}=$ $0.12-0.23)$. 
Tabla 2. Resultados de la razón de momios de las localidades agrupadas por su IDH, número de habitantes y densidad poblacional contra las proporciones de morbilidad, mortalidad y letalidad

\begin{tabular}{|c|c|c|c|}
\hline Localidades & Morbilidad & Mortalidad & Letalidad \\
\hline $\begin{array}{l}\text { Agrupadas por IDH } \\
\text { Muy alto y alto } \\
\text { Moderado y bajo }\end{array}$ & $\begin{array}{l}6.58(5.02-8.62) \\
0.15(0.12-0.19)\end{array}$ & $\begin{array}{c}7.82(6.05-10.13) \\
0.13(0.09-0.17)\end{array}$ & $\begin{array}{l}0.79(0.64-0.98) \\
1.26(1.02-1.56)\end{array}$ \\
\hline $\begin{array}{l}\text { Agrupadas por tamaño de población } \\
50000 \text { a } 1000000 \text { habitantes } \\
<2500-49999 \text { habitantes }\end{array}$ & $\begin{array}{l}3.24(2.56-4.10) \\
0.31(0.24-0.39)\end{array}$ & $\begin{array}{l}2.56(2.04-3.22) \\
0.39(0.31-0.49)\end{array}$ & $\begin{array}{l}0.65(0.51-0.82) \\
1.54(1.22-1.94)\end{array}$ \\
\hline $\begin{array}{l}\text { Agrupadas por densidad poblacional } \\
\text { Mayor que media nacional } \\
\text { Menor que media nacional }\end{array}$ & $\begin{array}{l}4.20(3.09-5.69) \\
0.24(0.18-0.32)\end{array}$ & $\begin{array}{l}5.98(4.28-8.36) \\
0.17(0.12-0.23)\end{array}$ & $\begin{array}{l}0.94(0.70-1.26) \\
1.06(0.79-1.43)\end{array}$ \\
\hline
\end{tabular}
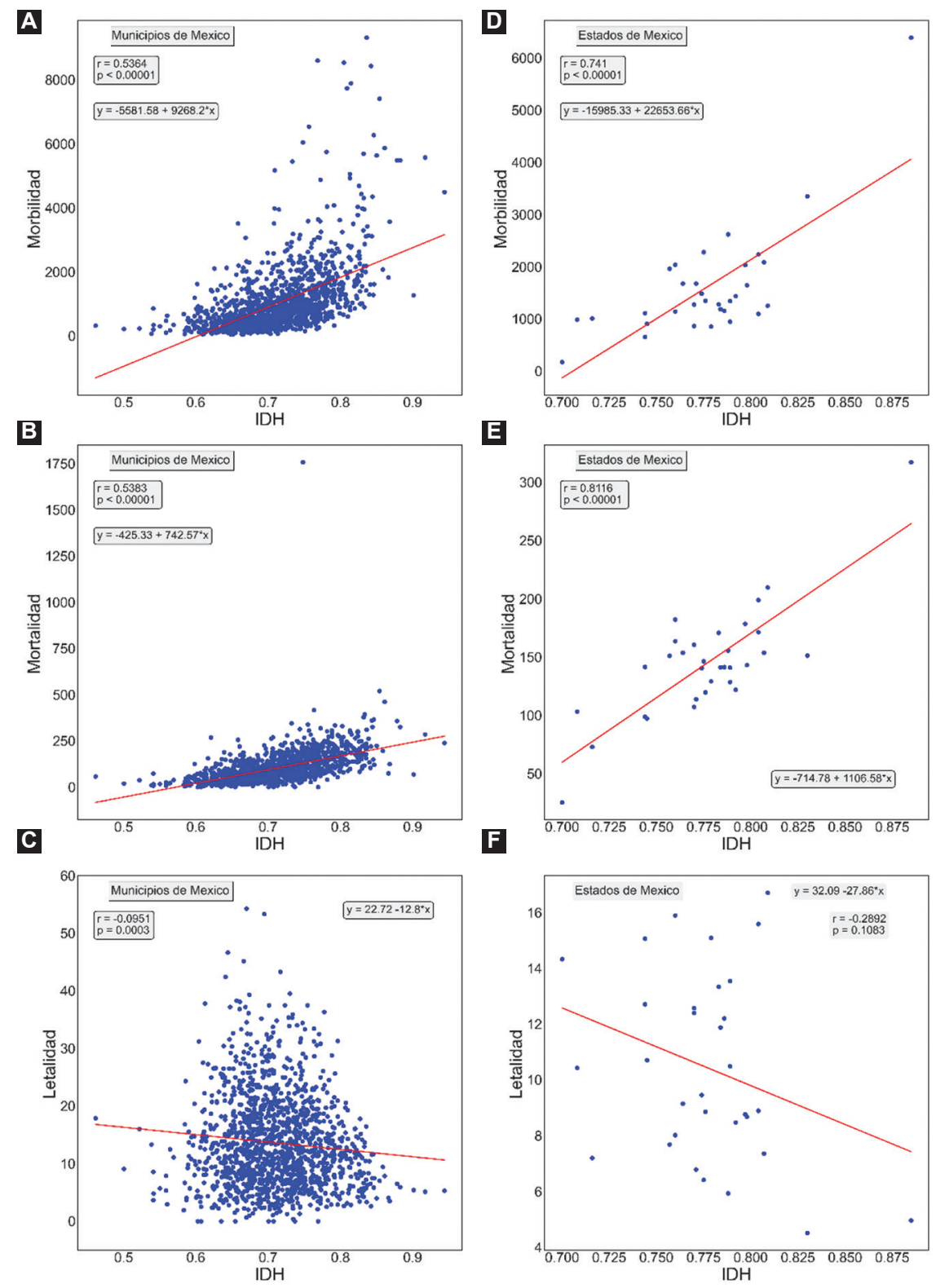

Figura 2. Relación de la morbilidad, mortalidad y letalidad con el índice de desarrollo humano (IDH) en los municipios (A-C) y estados (D-F) de México. 


\section{Agrupación jerárquica}

En las Figuras 3 y 4 se muestran los diagramas jerárquicos de las tasas de morbilidad, mortalidad, letalidad y las 16 medidas de pobreza multidimensional. Las relaciones más relevantes se observan en las ramas más internas del árbol, es decir, aquellas con las distancias euclidianas más cortas (eje X).

\section{Visualización bidimensional de los datos en el algoritmo $t-S N E$}

El resultado del algoritmo t-SNE para la reducción de dimensiones y visualización bidimensional se presenta en la Figura 5. Los municipios se agruparon de acuerdo con sus niveles de IDH. El tamaño de los símbolos se determinó por la letalidad; se observan símbolos muy grandes en el conjunto de municipios con IDH moderado y alto, no así en los municipios con IDH muy alto.

\section{Discusión}

Hasta la fecha de corte de este estudio, en $95.9 \%$ de los municipios del país se registró al menos un caso positivo de COVID-19. Los 100 municipios sin casos registrados se ubican en regiones montañosas, con baja densidad poblacional y con más de la mitad de su población con accesibilidad muy baja o baja a carretera pavimentada.

En este estudio, la morbilidad y mortalidad estuvieron relacionadas positivamente con el IDH de los municipios y estados de México. No obstante, la letalidad fue mayor en los municipios con más bajo desarrollo humano, lo que permite establecer que la enfermedad tiene efectos más severos en los estratos más desprotegidos de la población. Los análisis de riesgo fueron consistentes con lo anterior. En los municipios con alto y muy alto IDH se encontraron riesgos de morbilidad y mortalidad de seis hasta casi ocho veces mayores, pero esta condición socioeconómica brindó, a su vez, protección contra tener una letalidad por arriba de la media nacional. En contraste, los municipios con bajo y moderado IDH presentaron riesgo importante de letalidad superior a la media nacional. Esta tendencia se mantuvo cuando se analizaron los municipios de acuerdo con su tamaño de población: a menor tamaño aumentó significativamente el riesgo de alta letalidad.

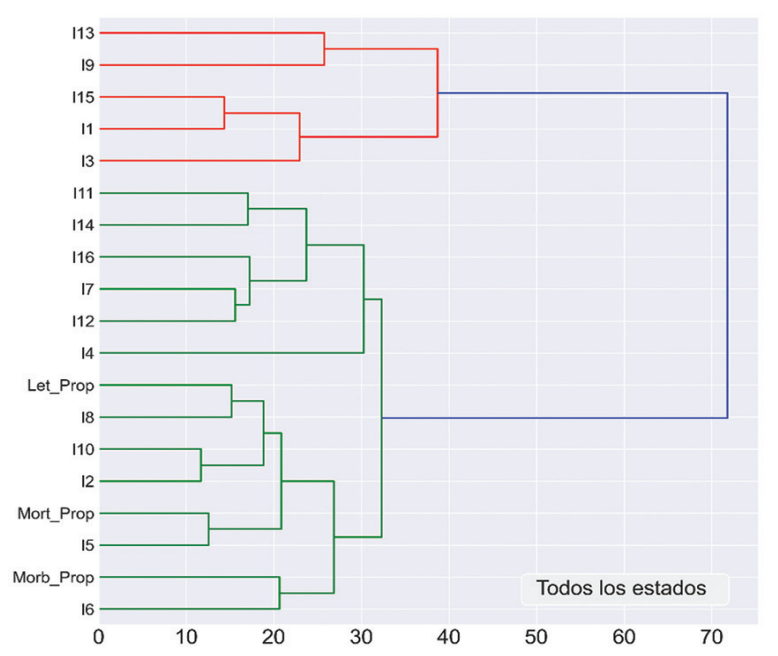

Figura 3. Análisis de clúster jerárquico de 19 variables (morbilidad, mortalidad, letalidad y 16 mediciones de pobreza multidimensional: I1 a (16), con el empleo de distancia euclidiana en todos los estados de México.

Del mismo modo, la densidad poblacional, definida como el número de habitantes por kilómetro cuadrado, desempeñó un papel determinante en el riesgo de presentar alta morbilidad y mortalidad. En el análisis de agrupación jerárquica, la tasa de morbilidad se relacionó directamente con la población no pobre y no vulnerable, mientras que la mortalidad se relacionó principalmente con la vulnerabilidad por ingresos. Este hallazgo resulta por demás revelador dado que la infección por SARS-CoV-2 puede tener mayor presencia en la población con mejor estatus socioeconómico, sin que necesariamente resulte en alta mortalidad, como sucede en las personas vulnerables por ingresos. La tasa de letalidad también se asoció predominantemente a la falta de servicios de salud $y$, a su vez, estas variables se relacionaron con otras como pobreza extrema, falta de calidad y espacio en la vivienda.

En la lucha contra COVID-19, el acceso a los servicios de salud es fundamental para el abordaje diagnóstico y terapéutico. El principal enfoque de las autoridades de salud debe ser extender la cobertura de los servicios de salud de primer nivel a las comunidades con mayor precariedad social, y aumentar el número de hospitales específicamente dedicados a la atención de los enfermos (hospitales COVID). Asimismo, en el corto o mediano plazo se deben mejorar las vías de comunicación de las comunidades para un traslado rápido y seguro a los centros de salud. Cabe señalar que actualmente, la atención a 

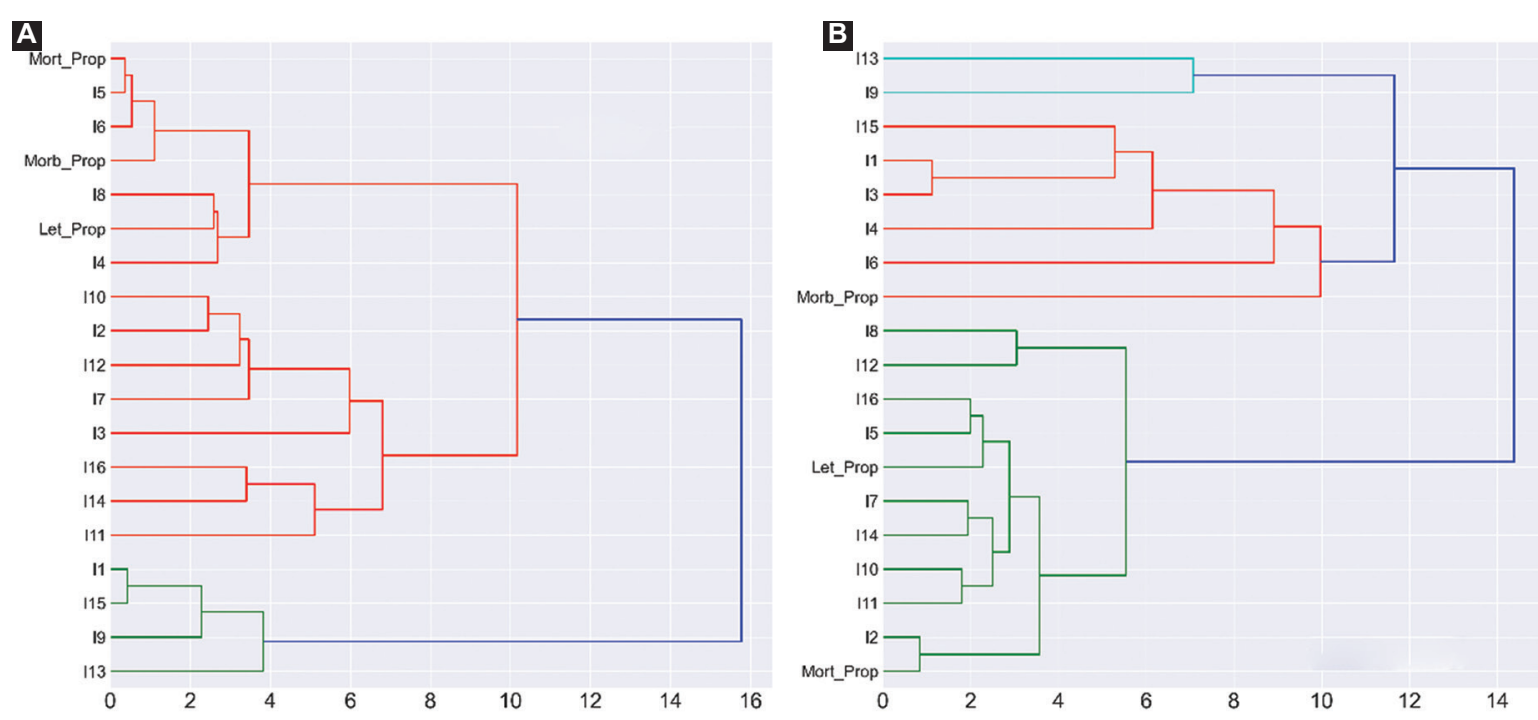

Figura 4. Análisis de clúster jerárquico para todos los municipios de México de diversos estados con los valores del índice de desarrollo humano fijo. A: percentil 5 de IDH, en el cual se ubican los municipios más pobres o con menor desarrollo. B: percentil 95 de IDH, en el cual se ubican los municipios con mayor desarrollo.

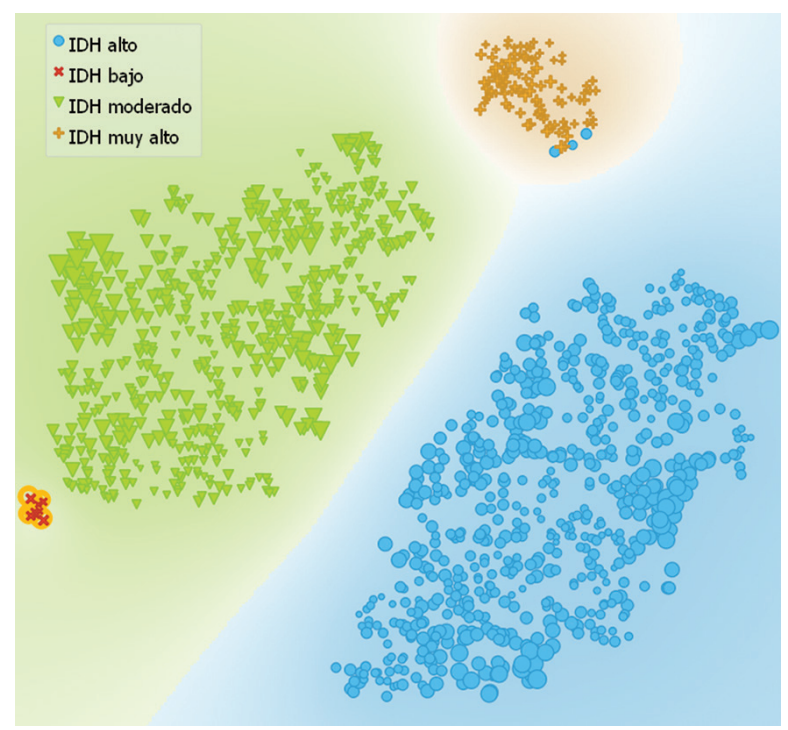

Figura 5. Visualización de los municipios mediante el método t-SNE conforme las tasas de morbilidad, mortalidad, letalidad, IDH y las 16 medidas de pobreza multidimensional. Los municipios se agrupan de acuerdo con sus niveles de IDH. Los puntos resaltados en amarillo corresponden a municipios rurales.

los sospechosos de COVID-19 es gratuita y no está restringida a quienes no tienen acceso a la seguridad social; sin embargo, es probable que los pacientes sin seguro médico no acudan a la atención privada por falta de recursos económicos, lo cual retrasa el diagnóstico y traslado a hospitales COVID, donde finalmente llegan con enfermedad avanzada y efectos irreversibles potencialmente mortales.
En los municipios con IDH más bajos de México, es decir del percentil 5 (Figura 4A), es posible identificar la relación directa entre la mortalidad y la vulnerabilidad por ingreso $y$, en segundo término, con la población no pobre y no vulnerable, la cual se conecta con la tasa de morbilidad. La letalidad está relacionada con la falta de acceso a servicios de salud y la vulnerabilidad por carencia social. En los municipios del percentil 95 (Figura 4B), la tasa de mortalidad está relacionada directamente con la proporción de pobreza extrema, y la morbilidad con las relaciones entre pobreza, pobreza moderada, vulnerabilidad por carencia social, ingreso inferior a la línea de bienestar y carencia por acceso a los servicios de salud. Finalmente, la letalidad está relacionada con la población con ingreso inferior a la línea de bienestar mínimo y las vulnerables por ingreso.

Con el algoritmo t-SNE se observó que la mayoría de las grandes zonas metropolitanas con IDH muy alto presentaron diversos niveles de letalidad, por ejemplo, los municipios fronterizos con los Estados Unidos de América, tales como Tijuana y Mexicali presentaron letalidad alta (16 a $20 \%$ ). Existen municipios con IDH moderado-alto y con letalidades superiores a $30 \%$ y de hasta $54 \%$, los cuales corresponden principalmente a ciudades medianas, pequeñas y algunas semirrurales.

\section{Conclusiones}

El presente estudio proporciona evidencia que sugiere que COVID-19 tiene efectos sobre la población 
con algún grado de vulnerabilidad y pobreza en México. La mayor transmisión ocurre en las zonas con alto y muy alto desarrollo económico, mientras que aquellas donde existen más pobreza y carencias sociales resultan más afectadas por la letalidad de la enfermedad. El principal indicador socioeconómico asociado a la letalidad es la falta de acceso a los servicios de salud, por lo que estos temas deben ser atendidos prioritaria y urgentemente por el Estado mexicano, para mitigar no solo la severidad de la actual pandemia sino para mejorar el estado general de salud de la población.

\section{Conflicto de intereses}

Los autores declaran que no tienen conflicto de intereses financieros 0 relaciones personales que pudieran haber influido en el trabajo reportado en este artículo.

\section{Financiamiento}

Los autores declaran no haber recibido financiamiento.

\section{Responsabilidades éticas}

Protección de personas y animales. Los autores declaran que para esta investigación no realizaron experimentos en seres humanos ni en animales.

Confidencialidad de los datos. Los autores declaran que en este artículo no aparecen datos de pacientes.

Derecho a la privacidad y consentimiento informado. Los autores declaran que en este artículo no aparecen datos de pacientes.

\section{Bibliografía}

1. Pérez-Tamayo R. Patología de la pobreza. México: El Colegio Nacional; 2016.

2. Wagstaff A. Pobreza y desigualdades en el sector de la salud. Rev Panam Salud Publica. 2002;11:316-326.

3. Bello-Chavolla OY, Bahena-López JP, Antonio-Villa NE, Vargas-Vázquez A, González-Díaz A, Márquez-Salinas A, et al. Predicting mortality due to SARS-CoV-2: a mechanistic score relating obesity and diabetes to COVID-19 outcomes in Mexico. J Clin Endocrinol Metab. 2020;105:dgaa346.

4. Denova-Gutiérrez E, López-Gatell H, Alomia-Zegarra JL, López-Ridaura R, Zaragoza-Jimenez CA, Dyer-Leal DD, et al. The association of obesity, type 2 diabetes, and hypertension with severe coronavirus disease 2019 on admission among Mexican patients. Obesity (Silver Spring). 2020:28:1826-1832

5. Kammar-GarcíaA, Vidal-MayoJJ, Vera-ZertucheJM, Lazcano-HernándezM, Vera-López O, Segura-Badilla O, et al. Impact of comorbidities in Mexican SARS-Cov-2-positive patients: a retrospective analysis in a national cohort. Rev Invest Clin. 2020;72:151-158.

6. Consejo Nacional de Evaluación de la Política de Desarrollo Social. La política social en el contexto de la pandemia por el virus SARS-CoV-2 (COVID-19) en México. Ciudad de México: Consejo Nacional de Evaluación de la Política de Desarrollo Social; 2020.

7. Bello-Chavolla OY, González-Díaz A, Antonio-Villa NE, Fermín-Martínez CA Márquez-Salinas A, Vargas-Vázquez A, et al. Unequal impact of structural health determinants and comorbidity on COVID-19 severity and lethality in older Mexican adults: looking beyond chronological aging. J Gerontol A Biol Sci Med Sci. 2021;76:e52-e59.

8. Pablos-Méndez A, Vega J, Aranguren FP, Tabish H, Raviglione MC. Covid-19 in Latin America. BMJ. 2020;370:m2939.

9. About Python [Internet]. EE. UU: Python Software Foundation; 2020.

10. Your data science toolkit [Internet]. EE. UU: Anaconda Inc.; 2020.

11. Consejo Nacional de Evaluación de la Política de Desarrollo Social. Informe de pobreza en los municipios de México 2015. México: Consejo Nacional de Evaluación de la Política de Desarrollo Social; 2018.

12. Peters DJ. Community susceptibility and resiliency to COVID 19 across the ruraldurban continuum in the United States. J Rural Health. 2020;36:446-456

13. Rosales-Rivera M, Díaz-González L, Verma SP. A new online computer program (BiDASys) for ordinary and uncertainty weighted least-squares linear regressions: case studies from food chemistry. Rev Mex Ing Quim. 2018;17:507-522.

14. Saha P. Decision support system to develop evidence-based policies for inequity reduction in maternal and child health care. J Health Manag. 2019;21:53-84.

15. Christiansen I. Health and development challenges: a nested typology of 123 developing countries. Int Crit Thought. 2019;9:297-313.

16. Demsar J, Curk T, Erjavec A, Gorup C, Hocevar T, Milutinovic M, et al. Orange: data mining toolbox in Python. J Machine Learning Res. 2013:14:2349-2353.

17. Secretaría de Salud/Gobierno de México [Internet]. México: Informe técnico diario COVID-19. 2020 Sep 05. 